\title{
Precise Time System Efficiency of a Frame Slotted Aloha based Anti-Collision Algorithm in an RFID System
}

\author{
Sunil Dhakal \\ Department of Computer Engineering, Chosun University. \\ Gwangju, South Korea \\ E-mail:sdhakal@chosun.kr \\ Seokjoo Shin (Corresponding Author) \\ Department of Computer Engineering, Chosun University. \\ Gwangju, South Korea \\ E-mail: sjshin@chosun.ac.kr
}

Received: March 13, 2013

Accepted: June 18, 2013

Published: June 30, 2013

DOI: $10.5296 /$ npa.v5i2.3373

URL: http://dx.doi.org/10.5296/npa.v5i2.3373

\begin{abstract}
The tag identification efficiency of a reader in an RFID system with Frame Slotted Aloha (FSA) based Anti-Collision Algorithm (ACA) can be maximized by selecting the optimal frame length with respect to the number of interrogating tags. Conventional analytical models that have been used widely to derive such an optimal frame length are inaccurate because they lack either precise characterization of the timing details of the underlying ACA or do not consider the physical layer capture effect. In this study, one of the most popular conventional analytical models has been extended not only to deliberate the exact timing details of the underlying ACA but also to consider the physical layer capture effect. Rigorous numerical analysis shows that the optimal frame length derived from the new extended model is precise, whereas that of from the conventional model deviates significantly from the true optimal value, particularly when the number of tags is high or the capture probability is low.
\end{abstract}

Keywords: RFID, Frame Slotted Aloha, Anti-Collision Algorithm, Capture Effect, Optimal Frame Size. 


\section{Introduction}

A simple RFID system consists of a single reader and multiple tags [1]. The reader activates the tags first and the tags send back their information. During the identification process, multiple RFID tags transmit their identification information arbitrarily to the designated reader using a shared wireless channel. If more than one tag transmits at a given time, the transmitted identification packets are assumed to be collided (if the received signal strength at the reader is below the capture threshold), and cannot be decoded correctly by the reader [2]. Therefore, designing and optimizing Anti-Collision Algorithms (ACAs) are fundamental to the effective use of RFID systems [3].

Electronic Product Code (EPC)-Global [4] Generation 2 (G2) has adopted a simply designed Frame Slotted Aloha (FSA) [5] as its ACA. One of the common approaches popularly used to optimize the system performance (tag identification efficiency) of RFID systems with FSA based ACA is to optimize a protocol parameter, such as frame duration or length, relative to the contending population size of the RFID tags.

Porta et. al. presented an analytical model to analyze the performance of FSA based ACA used in EPC-Global G2 RFID systems [6]. This model is useful not only for estimating the time system efficiency of the system, but also in deriving the optimal frame size with respect to the contending tag population. The model, however, has some limitations. First, one of the fundamental assumptions (equi-duration collision and successful slots) is not consistent with the EPC-Global G2 specifications. Another minor limitation is that the model does not consider the chances of a packet being captured when more than two tags transmit the identification packet simultaneously. Such a phenomenon is popularly known as capture effect (CE)[7].

In this paper, the aforementioned model [6] is extended. The extended model not only accurately differentiates the timing durations of the collisions and successful slots in accordance with the EPC-Global G2 specification, but also considers the physical layer capture effect. Rigorous numerical analysis shows that the so-called optimal frame length derived from the conventional analytical model deviates significantly from the true optimal value, particularly when the tag population is high or the capture probability is low.

The remainder of the paper is organized as follows: Section 2 briefly describes one of the relevant RFID standards focusing on its underlying ACA, whereas Section 3 presents an analytical model to estimate the tag identification efficiency of such an ACA. Section 4 presents the extended analytical model followed by some numerical results in Section 5 . Finally, Section 6 concludes the paper.

\section{Preliminaries}

This section provides the essential details specified in the RFID EPC-Global standard. The timing details of variable length slots were calculated with the specifications mentioned in the standard. In addition, an analytical model that can be used to calculate the tag 
identification efficiency of such ACA is reviewed.

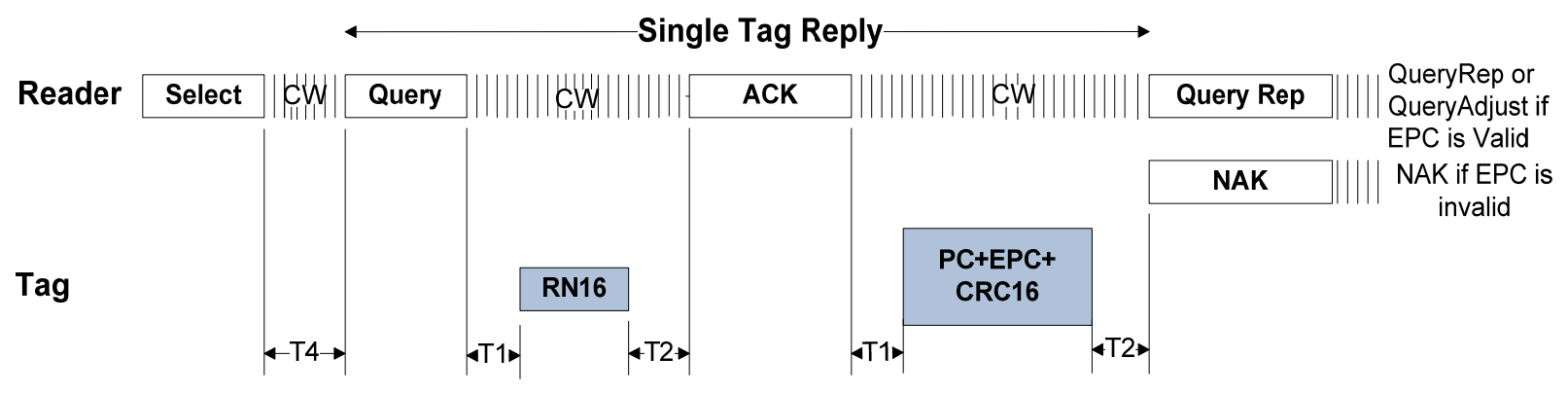

(a) Single Tag Reply (Success Slot)

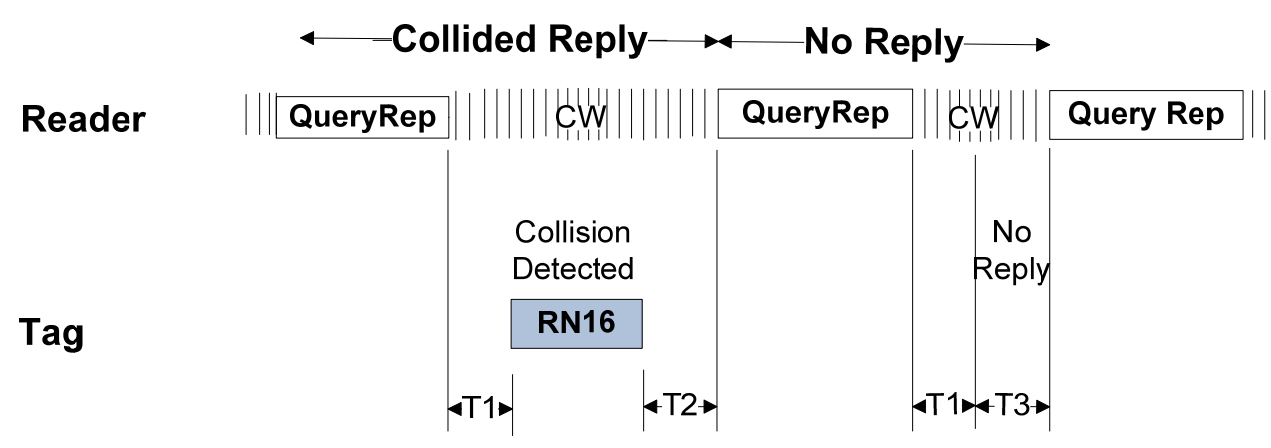

(b) Collided and No Tag Reply (Collided and Idle Slots)

Fig. 1. Success, Collision and Idle tag reply in EPC Global G2

\subsection{Frame Slotted Aloha in EPC Global}

The EPC-Global G2 protocol adopts the well-known FSA as its ACA. The fundamental principle of this popular protocol is that the fixed time frame determined and broadcasted by the reader is slotted into numerous discrete time intervals (slots) and each tag randomly selects any one slot to transmit its identification information. Therefore, independent transmission events from the tags can result in any of the following:

1. Idle (None of the tags transmit)

2. Success (Single tag transmits in a slot)

3. Collision (Two or more tags transmit in the same slot).

Fig. 1(a) shows the process during successful identification. The reader begins with the tag selection by transmitting the select command. The select command activates the tags and requests them to participate in the identification process. After the tags are activated, the reader sends the query command. The query command begins a new frame and requests each tag to generate a 16 bit random number (RN16) to select their transmitting slot. The tags maintain their slot counter according to the selected value. Tags decrease their slot counter at the end of each slot. As soon as the slot counter is zero, the tags reply with RN16. On the 
successful reception of RN16, the reader sends the Acknowledgement (ACK). The ACK command requests a tag to send its EPC. As a response, the tag sends its EPC along with other control messages, such as Protocol Control (PC) and 16 bit Cyclic Redundancy Check code (CRC16). If there is no error and the EPC code is valid then the reader begins the next slot by sending a Query Reply (QRep). In the case of error in EPC, the reader sends a Negative-ACK (NAK) command. The NAK requests the tag involved to transmit in the next frame.

Fig. 1 (b) shows the process during the collision and idle states. In the case of collision, the reader attempts to resolve the collision or without resolving the collision, it may send the NAK or QueryRep command. Similarly, if no tags reply for some threshold time, which is represented as T3 in Fig. 1(b), the reader assumes it to be idle slot and begins the next slot by issuing a new QueryRep command.

Table1. Timing Parameters used to calculate the time duration of each slot [4]

\begin{tabular}{|c|c|c|c|}
\hline Parameters Name & Symbol & Parameters Name & Symbol \\
\hline \hline QRep & 4 bits & EPC & 96 bits \\
\hline ACK & 18 bits & FS (Frame Sync) & $100 \mu \mathrm{s}$ \\
\hline CRC16 & 16 bits & T1 & $125 \mu \mathrm{s}$ \\
\hline RN16 & 16 bits & T2 & $62.5 \mu \mathrm{s}$ \\
\hline PC & 16 bits & T3 & $62.5 \mu \mathrm{s}$ \\
\hline P (preamble) & 6 bits & Data Rate & $40 \mathrm{kbps}$ \\
\hline
\end{tabular}

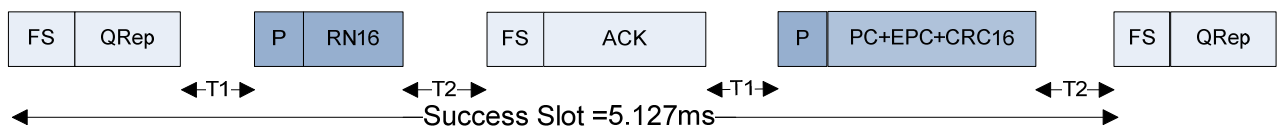

(a) Success Slot Duration

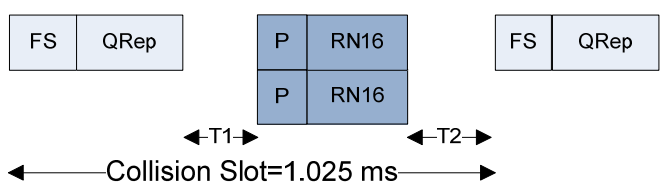

(b) Collision Slot Duration

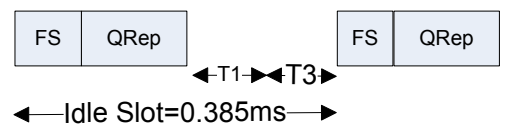

(c) Idle Slot Duration

Fig. 2. Success, Collision and Idle slot duration in EPC Global G2 


\subsection{Timing Details in EPC Global}

In the EPC-Global G2 protocol, each command, data symbol, preamble and turnaround transmission period between the reader and tag are comprised of certain time duration, as shown in Fig. 1 (a) and (b). T1 and T2 are the turnaround time. T1 is the time interval from a reader's transmission to the tag's response, whereas T2 is the time interval from the tag's response to the reader's next transmission. T3 is the idle time interval, as mentioned above. T4 is the time between the select command and the first query command. From the parameters presented in Table 1 and the timing diagrams in Fig. 2(a), (b) and (c), the slot time duration for success, collision and idle events can be calculated as follows:

$$
\begin{gathered}
T_{S}=T_{Q R E P}+2 T_{1}+T_{R N 16}+2 T_{2}+T_{A C K}+T_{(P C+E P C+C R C)}=5.127 \mathrm{~ms} \\
T_{C}=T_{Q R E P}+T_{1}+T_{R N 16}+T_{2}=1.025 \mathrm{~ms} \\
T_{I}=T_{Q R E P}+T_{1}+T_{3}=0.385 \mathrm{~ms}
\end{gathered}
$$

\subsection{Slot Distribution in EPC Global}

In EPC-Global G2, each tag can access the channel only once in a single frame. The collided tags need to wait for another frame to transmit again. In any arbitrary frame, if the frame size of $N$ slots is used for $n$ number of tags, then $r$ number of tags in one slot is distributed binomially with the parameters $n$ and $1 / N[5]$ :

$$
B_{n, 1 / N}(r)=\left(\begin{array}{l}
n \\
r
\end{array}\right)(1 / N)^{r}(1-1 / N)^{n-r} .
$$

The $r$ number of tags in a particular slot is called the occupancy number of a slot. Therefore, the expected number of slots with occupancy $r$ is given by the following equation:

$$
a_{r}^{N, n}=N B_{n, 1 / N}(r)=N\left(\begin{array}{l}
n \\
r
\end{array}\right)(1 / N)^{r}(1-1 / N)^{n-r} .
$$

When $r=1$, the particular slot is filled with precisely one tag. Therefore, the number of successful slots $(S)$ out of $\mathrm{N}$ slots in a frame is given by

$$
S=a_{1}^{N, n}=N B_{n, 1 / N}(1)=n(1-1 / N)^{n-1} .
$$

From (5), the number of idle slots (I) can also be expressed as

$$
I=a_{0}^{N, n}=N B_{n, 1 / N}(0)=N(1-1 / N)^{n} .
$$

From (5), (6) and (7), the number of collision slots $(C)$ can be written as

$$
C=a_{\geq 2}^{N, n}=N-S-I
$$




\section{Related Works}

To maximize the performance of RFID systems, wide range of ACAs has been studied and their performance metric has been modeled accordingly. However, no existing protocols are optimized using the precise characterization of the duration of slots of underlying ACA. This section presents two conventional performance evaluation metrics, named System Efficiency (SE) and Time System Efficiency (TSE). In addition, how these metrics are used to derive the optimal frame size is also reviewed. The SE only considers the number of slot events but lacks to differentiate the duration of different slot events. Further, the TSE considers the duration of slot events but still lacks the precise characterization of success and collision slot events.

\subsection{System Efficiency}

$\mathrm{SE}$ is one of the most common evaluation metrics used to evaluate the performance of the RFID ACAs. Metric SE simply considers the number of successful slots out of the total number of slots, as given in the following equation [8]

$$
S E=\frac{S}{N}=\frac{S}{I+S+C}
$$

From (6) and (9)

$$
S E=\frac{n(1-1 / N)^{n-1}}{N} .
$$

The optimal $\mathrm{N}$ (i.e. $\mathrm{N}^{*}$ ) that maximizes $\mathrm{SE}$ can be calculated easily by solving the following differential equation:

$$
\frac{d(S E)}{d N}=0 .
$$

Solving (11) gives

$$
N^{*}=n
$$

Equation (12) indicates that SE is maximized when the frame size is selected to be equal to the tag population.

\subsection{Time System Efficiency}

The SE calculated in the above subsection is not precisely correct because it assumes that the event duration for successful, collision and idle events are equal. Nevertheless, as described in the previous section, those event durations are not equal. Porta et. al. differentiated the duration of idle and successful or collision events and reported that information while calculating SE, which resulted in a new metric called TSE. Porta et. al. shows that the idle events are not involved in the transmission of tag ID's as shown in Fig. 1(b), they are shorter than the success and collision event durations. Thus, during evaluation 


\section{Macrothink}

the idle slot duration has been normalized with the length of success slots with the multiplicative factor $\beta$ (which value is $\mathrm{T}_{\mathrm{I}} / \mathrm{T}_{\mathrm{S}}$ ), as expressed in the following equation:

$$
T S E=\frac{S}{\beta I+S+C} .
$$

The optimal frame size $\left(\mathrm{N}^{*}\right)$ can be calculated by solving the following differential equation:

$$
\frac{d(T S E)}{d N}=0
$$

The maximum value is achieved when

$$
(\beta-1)(1-1 / N)^{n}+n\left(1-\frac{1}{N}\right)+(1-n)=0 .
$$

Solving (15) and finding the roots of $\mathrm{N}$ for different sets of tags, the optimal frame size $\mathrm{N}^{*}$ for $\mathrm{n}$ number of tags can be expressed as

$$
N^{*}=4.406 \times n-1 .
$$

Equation (16) suggests that TSE is maximized when the frame size is selected to be 4.4 times larger than the tag population.

\section{New Optimal Frame Size Calculation}

Although TSE considers different successful/collision and idle slot event durations, it does not differentiate the duration of the successful and collision events. For EPC-Global G2, however, those durations are different, as can be noted in (1) and (2). Therefore, the TSE in (9) has been revised to formulate a Precise TSE (PTSE) as follows:

$$
P T S E=\frac{S}{\beta I+S+\alpha C},
$$

where $\alpha$ is a multiplication factor $\left(\mathrm{T}_{\mathrm{C}} / \mathrm{T}_{\mathrm{S}}\right)$.

Reconfiguring (17) as

$$
P T S E=\frac{S}{I(\beta-\alpha)+S(1-\alpha)+\alpha N}
$$

Substituting the values of S and I from (6) and (7) in (18) gives

$$
\text { PTSE }=\frac{n(1-1 / N)^{n-1}}{N(1-1 / N)^{n}(\beta-\alpha)+n(1-1 / N)^{n-1}(1-\alpha)+\alpha N} .
$$


The optimal frame size $\left(\mathrm{N}^{*}\right)$ can be then obtained by differentiating PTSE with respect to $\mathrm{N}$ as follows:

$$
\frac{d(P T S E)}{d N}=0
$$

Upon simplifying (20), the maximum PTSE can be achieved when

$$
\frac{(\beta-\alpha)}{\alpha}\left(1-\frac{1}{N}\right)^{n}+n \alpha\left(1-\frac{1}{N}\right)+\alpha(1-n)=0 .
$$

Solving (21) and finding the roots of $\mathrm{N}$ for different set of tags, the relation between $\mathrm{N}^{*}$ and the number of tags $n$, can be obtained as follows:

$$
N^{*}=1.46 \times n-1
$$

All the calculations presented thus far are based on the assumption that the simultaneous transmission of RFID tag information from more than two tags results in a collision. On the other hand, this assumption is not always true because even in the case of simultaneous transmissions, a transmission may be decoded well at the reader if its signal strength is above a given threshold (known as the capture threshold). Such a phenomenon is known as CE.

To consider the CE in PTSE, the PTSE in (17) is revised as

$$
P T S E_{C E}=\frac{S+\gamma(\alpha C)}{I(\beta-\alpha)+S(1-\alpha)+\alpha N} .
$$

Note that the mean capture probability $(\gamma)$ in (23) can be calculated using the following expression [9]:

$$
\gamma=\sum_{i=2}^{n} p_{c a p}(i) p_{c o l}(i)
$$

where $P_{c a p}(i)$ is the capture probability for $i$ collided tags and $P_{c o l}(i)$ is the probability of $i$ tags colliding in a given slot.

Therefore, the optimal frame size can be calculated by differentiating PTSE $E_{C E}$ with respect to $\mathrm{N}$, as shown below:

$$
\frac{d\left(P T S E_{C E}\right)}{d N}=0 .
$$

Solving (25), the maximum $P T S E_{C E}$ can be achieved when the following equation holds:

$$
\left(1-\frac{1}{N}\right)[(\beta-\alpha)+\gamma \alpha(1-\beta)]+[\gamma \alpha \beta+n \alpha(1-\gamma)]\left(1-\frac{1}{N}\right)+\alpha(1-\gamma)(1-n)=0 .
$$

Using the values of $\alpha=0.2$ and $\beta=0.075$ and solving (26), different optimal frame sizes 
can be obtained for different capture probabilities $(\gamma), \gamma \in[0,1]$.

Table 2. Optimal Frame Size with Different Capture Probabilities.

\begin{tabular}{|l|c|c|}
\hline $\begin{array}{c}\text { Average Capture } \\
\text { Probability }(\boldsymbol{\gamma})\end{array}$ & $\begin{array}{c}\text { Optimal Frame Size } \\
\left(\mathbf{N}^{*}\right)\end{array}$ & $\begin{array}{c}\text { Precise Time System } \\
\text { Efficiency (PTSE) }\end{array}$ \\
\hline 0 & $1.459 \mathrm{n}$ & 0.8375 \\
\hline 0.1 & $1.387 \mathrm{n}$ & 0.8432 \\
\hline 0.2 & $1.312 \mathrm{n}$ & 0.8513 \\
\hline 0.3 & $1.231 \mathrm{n}$ & 0.8599 \\
\hline 0.4 & $1.146 \mathrm{n}$ & 0.8694 \\
\hline 0.5 & $1.053 \mathrm{n}$ & 0.8798 \\
\hline 0.6 & $0.951 \mathrm{n}$ & 0.8916 \\
\hline 0.7 & $0.835 \mathrm{n}$ & 0.9052 \\
\hline 0.8 & $0.696 \mathrm{n}$ & 0.9219 \\
\hline 0.9 & $0.509 \mathrm{n}$ & 0.9449 \\
\hline 1 & 1 & 1 \\
\hline
\end{tabular}

As evident from Eq. (22), the maximum TSE of an RFID system (without considering $\mathrm{CE})$ can be achieved by fixing the frame length to almost 1.46 times larger than the contention population size. On the other hand, this calculation does not hold when the capture probability increases. Table 2 shows how the capture probability plays an important role in the optimal frame size estimation and how this optimal frame size calculation is an important feature that should be considered to obtain the optimal time system efficiency.

Note that when $\gamma=1$, which corresponds to the case of perfect capture (i.e., one tag is always captured regardless of the number of tags involved in the collision), the optimal frame size is 1 and theoretically system efficiency is $100 \%$.

\section{Analytical Results and Discussion}

In the realistic RFID environments, the variable length slots and capture effect phenomenon are widely used and we have developed our analytical model based on these factors. This section presents the results obtained from the proposed analytical model and explains how the results of the optimal frame size calculation are more precise than that of the conventional model.

To compare the proposed analytical model with the conventional model, two different schemes used in FSAs were considered: static and dynamic. We have developed a system level simulator using Matlab. The tags (10-1000) are randomly distributed within the interrogation zone of the reader. In the static FSA, the reader broadcasts the frame length once and all the tags use the same frame length throughout. On the other hand, in the dynamic FSA, the reader periodically estimates the tag population, calculates the optimal 
frame size and broadcasts the calculated frame size. For the static scheme, the frame size consisting of 128 contention slots is considered, whereas for the dynamic one, the frame size (relative to tag population) derived from the proposed analytical model and conventional model are taken into account.

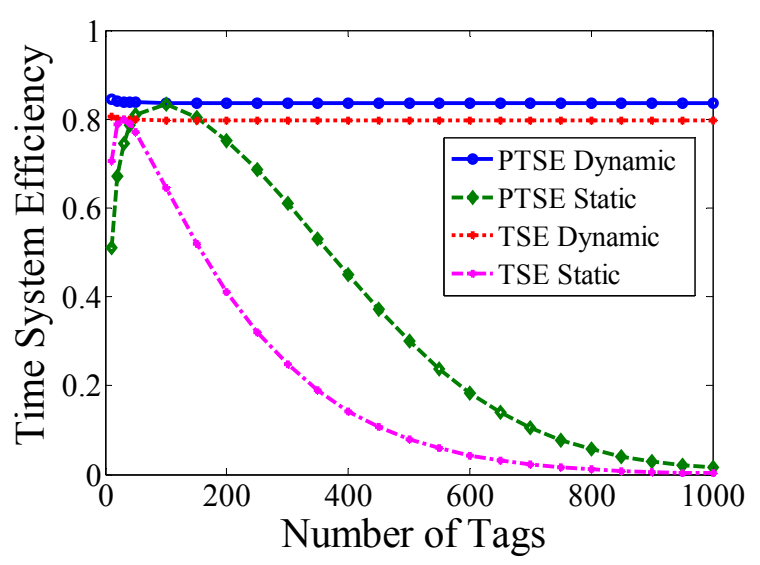

(a) $\gamma=0$ (Without Capture Effect)

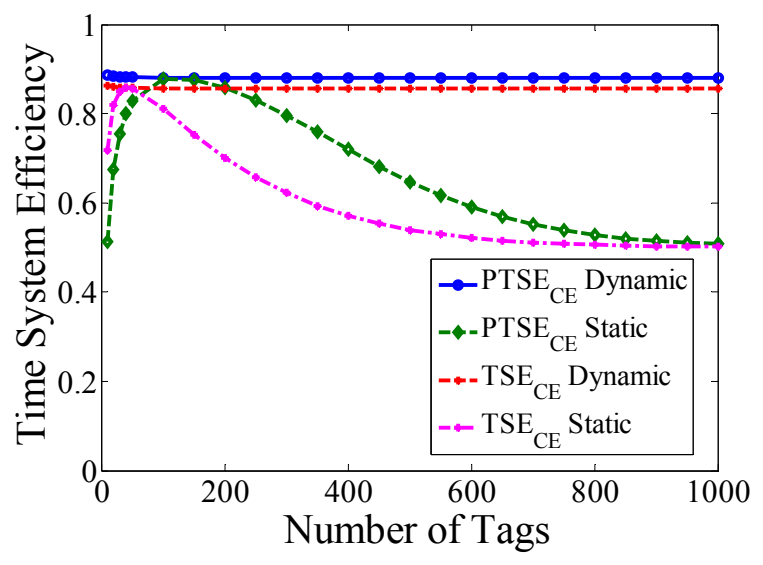

(c) $\gamma=0.5$

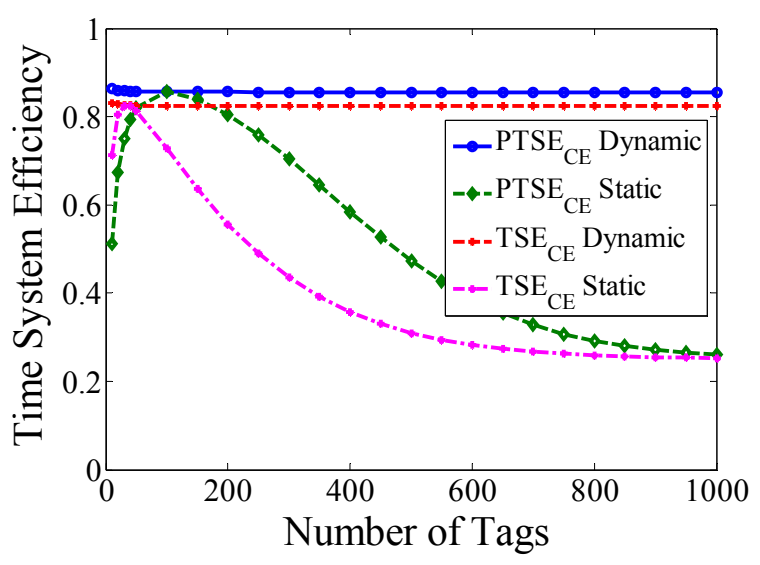

(b) $\gamma=0.25$

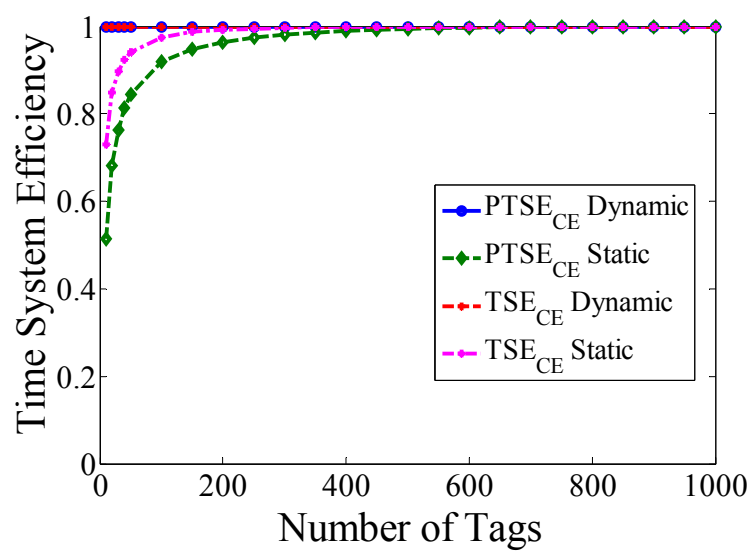

(d) $\gamma=1$

Fig. 3. Comparison of the Time System Efficiency of the Static and Dynamic FSA in RFID Systems

Fig. 3 (a) shows the time system efficiency of a static FSA and dynamic FSA calculated using the proposed model and the conventional model for the increasing number of interrogating tags. From the figure, it is evident that the conventional model overestimates the time system efficiency of the static FSA based RFID system when the tag population is low $(<$ half the frame size) i.e., when the tag population is less than half of the frame size (128 slots) the obtained TSE is higher than the PTSE. Furthermore, the figure illustrates that the TSE is lower than the PTSE when the tag population increases which clarifies that the conventional model underestimates the time system efficiency when the tag population is high. From the same figure, it is also evident that the conventional model underestimates ( $4 \%$ ) the time system efficiency of dynamic FSA based RFID systems. These results verify that optimal frame size value has been overestimated in literature [6] which is about 3 times higher than the optimal frame size value calculated using PTSE. 
Note that results presented in Fig. 3 (a) are for an ideal case, considering the no capture effect, i.e. capture probability $\gamma=0$ and the obtained optimal TSE is $84 \%$. However, in practical RFID systems, there is a certain capture probability less than one. Fig. 3 (b) and Fig. 3 (c) show that when the capture probability increases, the estimation error of the conventional model begins to decrease because the number of collisions in the system is reduced due to capture effects. Therefore, the effect of an incorrect characterization of collision slots cannot magnify the results significantly. Also, the figures illustrate that the deviation of the conventional model decreases when the capture probability increases. The optimal TSE also increases when the capture probability increases.

When the capture effect is 1 (the hypothetical case in Fig. 3 (d)), the time system efficiency estimated using the proposed model and conventional model is the same $(100 \%)$ for the dynamic because no collisions exist in the system.

\section{Conclusion}

This paper reported an accurate analytical model to estimate the tag identification efficiency of the FSA based anti-collision algorithm in an RFID system. The present model improves the conventional model not only by accurately differentiating the timing durations of success, collision and idle events but also by considering the physical layer capture phenomenon. Through detailed numerical analysis, we have shown that the conventional model overestimates the time system efficiency of the static FSA based RFID system when the tag population is low and vice versa. In addition, the conventional model underestimates the time system efficiency of the dynamic FSA based RFID system regardless of the tag population size. We intend to analyze the protocols using different performance metrics and perform testbed experiments in our future work.

\section{Acknowledgement}

This research was supported by Basic Science Research Program through the National Research Foundation of Korea (NRF) funded by the Ministry of Education (2012R1A1A2006327).

\section{References}

[1] Want, R. "An introduction to RFID technology," IEEE Pervasive Computing, Vol. 5, Issue 1, pp. 25-33, Jan. 2005. http://dx.doi.org/10.1109/MPRV.2006.2

[2] Finkenzeller, K., RFID Handbook: Fundamentals and Applications in Contactless Smart Cards and Identification, John Wiley \& Sons, Inc., 2003.

[3] Chen, Y. C., Yeh, K. H., Lo, N., Li, Y., \& Winata, E.," Adaptive collision resolution for efficient RFID tag identification," EURASIP Journal on Wireless Communications and Networking, no. 1, pp. 1-14, Oct. 2011. http://dx.doi.org/10.1186/1687-1499-2011-139 
[4] EPC Radio-Frequency Identification Protocols Class-1 gen-2 UHF RFID Protocol for communication at 860 MHz-960 MHz, Version 1.2.0, http://www.gs1.org/gsmp/kc/epcglobal/uhfc1g2, 2004-208 EPC global Inc.

[5] Vogt, H., "Efficient Object identification with passive RFID tags," First International Conference on Pervasive Computing, vol. 2414, pp. 98-113, Apr. 2002. http://dx.doi.org/10.1007/3-540-45866-2_9

[6] La Porta, T. F., Maselli, G., \& Petrioli, C., "Anti-Collision protocols for single-reader RFID Systems: temporal analysis and optimization," IEEE Transections on Mobile Computing, Vol. 10, Issue 2, pp. 267-279, Feb. 2011. http://dx.doi.org/10.1109/TMC.2010.58

[7] Zorzi, M., \& Rao, R. R., "Capture and retransmission control in mobile radio," IEEE Journal on Selected Areas in Communications, Vol. 12, Issue 8, pp. 1289-1298, Oct. 1994. http://dx.doi.org/ 10.1109/49.329345

[8] Lee, S. R., Joo, S. D., \& Lee, C. W., “An enhanced dynamic framed slotted aloha algorithm for RFID tag identification," MOBIQUITOUS '05, Washington, DC, USA, pp. 166-172, July 2005. http://dx.doi.org/10.1109/MOBIQUITOUS.2005.13

[9] Li, B., \& Wang, J., "Efficient anti-collision algorithm utilizing the capture effect for ISO 18000-6C RFID protocol," IEEE Communication Letters, Vol. 15, Issue 3, pp. 352-354, Mar. 2011. http://dx.doi.org/10.1109/LCOMM.2011.011311.101332

\section{Copyright Disclaimer}

Copyright reserved by the author(s).

This article is an open-access article distributed under the terms and conditions of the Creative Commons Attribution license (http://creativecommons.org/licenses/by/3.0/). 